\title{
Quantum Key Distribution using Two Coherent States of Light and their Superposition
}

\author{
Yoshihiro Nambu, Akihisa Tomita, Yoshie Chiba-Kohno, and Kazuo Nakamura \\ Fundamental Research Laboratories, NEC Corporation, \\ 34 Miyukigaoka, Tsukuba, Ibaraki 305-8501, Japan
}

(September 20, 2018)

\begin{abstract}
Quantum mechanical complementarity ensures the security of the keydistribution scheme reported by Brassard and Bennet in 1984 (BB84), but does not prohibit use of multi-photons as a signal carrier. We describe a novel BB84 scheme in which two nearly orthogonal coherent states carry the key, and the superposition of these states (cat states) protects the communication channel from eavesdropping. Information leakage to eavesdroppers can be determined from the visibility of the interferential fringes in the distribution of the outcome when a certain quadrature component is measured through homodyne detection. The effect of channel loss and detector inefficiency is discussed.
\end{abstract}

23.23.+x, 56.65.Dy

Typeset using REVTEX 


\section{INTRODUCTION}

The quantum key distribution (QKD) protocol provides a way for two remote parties (traditionally known as Alice and Bob) to share a secure random key by communicating over an open channel [1 5]. Alice and Bob publicly communicate over a quantum channel and then exchange messages over a classical channel that can be monitored but not tampered with by an eavesdropper (Eve). Quantum mechanics ensures that any activities of potential eavesdroppers can be detected. Even if some eavesdropping is found, Alice and Bob can further process the obtained key (the raw key) to extract a safe but much shorter key (the final key) by using a classical method of error correction (a reconciling protocol) and private amplification [6,7]. A secure message of equal length to the final key can be transmitted over the classical channel by conventional encryption methods such as the one-time pad method 8]. The security of the encrypted communication depends directly on the security of the final key.

Among the protocols proposed so far, the four-state scheme, usually referred to as the BB84 protocol [2], is claimed to be provably secure under the assumption that Alice uses a perfect single-photon source [9]. In this protocol, Alice and Bob use two conjugate bases (say, a rectilinear basis, + , and a diagonal basis, $\times$ ) for the polarization of a single photon. In basis + , they use two orthogonal states $\left|0_{+}\right\rangle$and $\left|1_{+}\right\rangle$to encode logical "0" and " 1 ", respectively, and in basis $\times,\left|0_{\times}\right\rangle\left(=(1 / \sqrt{2})\left[\left|0_{+}\right\rangle+\left|1_{+}\right\rangle\right]\right)$and $\left|1_{\times}\right\rangle\left(=(1 / \sqrt{2})\left[\left|0_{+}\right\rangle-\left|1_{+}\right\rangle\right]\right)$. Alice transmits a random sequence of these states through their quantum channel and Bob measures each state with a basis randomly chosen from $\{+, \times\}$. After transmission, the basis is revealed, which enables Bob to discard the data that Alice and Bob used a different basis to encode and decode and that provide inconclusive results to Bob. The remaining data, which is called the sifted key [10], should agree for Alice and Bob and yield conclusive results for Bob.

The key idea of the BB84 protocol is that simultaneous measurements of non-commuting observables for a single quanta are forbidden by quantum mechanical complementarity. For these non-commuting observables, the measurement of one observable made on the eigenstate of another observable inevitably introduces disturbance to the state because of the back reaction of the measurement. Since Eve has no a priori information about the randomly chosen bases of each bit in the sifted key, she is forced to guess which observable to measure for each photon. On average, half the time Eve will guess wrong and thus introduce a disturbance into the state. The disturbance can be detected as a bit error by comparing parts of the sifted key.

The theoretical QKD schemes that have been proven secure against a wide class of attacks have involved the transmission of a single particle that is subject to quantum mechanics. On the other hand, there has been growing interest among researchers on quantum information processing using multi-photon states [11,12]. Several authors have extended this idea and have recently proposed a QKD scheme that uses multi-photon states as a quantum carrier [13 15. All these authors used squeezed states, in which the key data are encoded on continuous, conjugate observables of the field quadrature components. Hillery further suggested that any nonclassical field state is useful for quantum information processing and communication [14]. In this paper, we show that quantum mechanics allows use of multi-photon states as a signal carrier in the BB84 protocol, and provide another example that supports Hillery's suggestion by showing that a secure BB84 protocol can be constructed by using 
two nearly orthogonal coherent states and the superposition of these states (cat states).

The organization of this paper is as follows. Section II reviews the BB84 protocol. The connection between the protocol and the information exclusion principle proposed by Hall [16] is discussed and a comprehensive explanation of the principle of the BB84 protocol is given. The importance of an exact determination of information leakage to eavesdroppers is stressed and what is required for the BB84 protocol is explained. Section III is devoted to the main subject of this paper. The basic idea and the protocol of the QKD scheme using two coherent states and their superposed state are presented, and the principle and security of this scheme are discussed. Section IV is mainly devoted to discussion of the effect of channel loss and detector inefficiency for both the present scheme and the conventional scheme. In Sec. $\nabla$, we summarize the main results of the paper.

\section{BB84 PROTOCOL}

The BB84 protocol can most clearly be understood in terms of the information exclusion principle [16]. This principle provides an information-theoretic description of quantum complementarity and imposes an upper bound on the sum of the information gain obtained from observation of complementary observables in a quantum ensemble. Consider two observables $A$ and $B$ of a quantum system with an $N$-dimensional Hilbert space. They are said to be complementary if their eigenvalues are nondegenerate, and the overlap of any two normalized eigenvectors $\left|a_{j}\right\rangle$ of $A$ and $\left|b_{j}\right\rangle$ of $B$ satisfy $\left|\left\langle a_{i} \mid b_{j}\right\rangle\right|=1 / \sqrt{N}$; therefore, the eigenstates of $A$ are equally weighted superpositions of the eigenstates of $B$, and vice versa. Thus, when the system is in an eigenstate of $A$, all possible outcomes of a measurement of $B$ are equally probable; i.e., precise knowledge of the measured value of one observable implies maximal uncertainty of the measured value of the other. In such a case, an operator $B$ is the generator of shifts in the eigenvalue of any eigenstate of $A ; \exp (i B l)\left|a_{j}\right\rangle=\left|a_{(j+l) \bmod N}\right\rangle$, and vice versa, $\exp (i A m)\left|b_{j}\right\rangle=\left|b_{(j-m) \bmod N}\right\rangle$ [17,18].

Hall proved an inequality concerning information gain obtained by the measurement of complementary observables $A$ and $B$ on a system in arbitrary state $\rho$. Let $\rho$ be a state of an given ensemble which is prepared with a priori probability $p_{i}$ in the known state $\rho_{i}$, so $\rho=\sum_{i} p_{i} \rho_{i}$. The initial entropy of the system is $H_{\text {int }}=H(\rho)=-\sum_{i} p_{i} \log _{2} p_{i}$ (in bits). Given the conditional probability $P\left(a_{j} \mid \rho_{i}\right)=\operatorname{tr}\left(\rho_{i} A_{j}\right)$ for obtaining outcome $a_{j}$ when measuring an observable $A$ of the state prepared in $\rho_{i}$, where $A_{j}=\left|a_{j}\right\rangle\left\langle a_{j}\right|$, we can compute the a posteriori probability $Q\left(\rho_{i} \mid a_{j}\right)$ for preparation $\rho_{i}$ by Bayes's theorem as $Q\left(\rho_{i} \mid a_{j}\right)=P\left(a_{j} \mid \rho_{i}\right) p_{i} / q_{j}$, where $q_{j}=\sum_{i} P\left(a_{j} \mid \rho_{i}\right) p_{i}$ is the a priori probability for the occurrence of outcome $a_{j}$. After the measurement, the average entropy (in bits) becomes $H_{\text {fin }}=H(\rho \mid A)=-\sum_{j} q_{j} \sum_{i} Q\left(\rho_{i} \mid a_{j}\right) \log _{2} Q\left(\rho_{i} \mid a_{j}\right)$. The average information gain (in bits) is $I(\rho ; A) \equiv H_{\text {ini }}-H_{\text {fin }}=H(\rho)-H(\rho \mid A)=-\sum_{i} p_{i} \log _{2} p_{i}+\sum_{j} q_{j} \sum_{i} Q\left(\rho_{i} \mid a_{j}\right) \log _{2} Q\left(\rho_{i} \mid a_{j}\right)$; 19,20 this is also called the Shannon mutual information. Hall proved that the inequality

$$
I(\rho ; A)+I(\rho ; B) \leq 2 \log _{2} N \xi=\log _{2} N
$$

holds for the measurement of complementary observables $A$ and $B$ on a system in arbitrary state $\rho$, where $\xi=\max \left|\left\langle a_{j} \mid b_{j}\right\rangle\right|=1 / \sqrt{N}$ [16]. When $N=2$, inequality (2.1) means that the recoverable information can never exceed the maximal von Neumann entropy $\left(S_{\max }=1\right)$ 
bit of the system, which depends only on the dimension - the number of distinguishable pure states - of the Hilbert space in which the signal states lie. Inequality (2.1) states that the information gain corresponding to the measurement of an observable can be maximized only at the expense of the information gains corresponding to the measurement of the complementary observable. Hall named inequality (2.1) the information exclusion principle and showed that it is closely related to Heisenberg's uncertainty principle and Bohr's complementary principle [16].

To see how the information exclusion principle relates to the BB84 protocol, let us briefly review the optimal eavesdropping strategy within an individual-attack scheme in which each signal carrier sent by Alice is independently subject to eavesdropping. In this strategy, Eve lets a probe of arbitrary dimensions interact with each signal carrier independently. As a result, each of her probes is correlated to a transmitted state and its partial information is imprinted onto the probe. She then delays her measurement and keeps the quantum information in her probes until she learns the bases used by Alice and Bob from their public announcement. She finally tries to extract as much information as possible about the transmitted states by measuring her probes. To avoid revealing herself in too straightforward a manner by introducing different error rates in the different bases (because the error rate should be independent of the basis if the errors are due to a random process), Eve applies a symmetric eavesdropping strategy that treats the two bases on an equal footing. This strategy has been shown to require a two-qubit probe - i.e., a quantum system with a four-dimensional Hilbert space - and to be optimal by Fuchs [21]. He proved that the joint unitary operation $U$ acting on the Hilbert space of the carrier and probe is a state-dependent optimal quantum-cloning process [22 24] that is given by

$$
\begin{aligned}
|\psi\rangle\left|0_{x}\right\rangle & \rightarrow U|\psi\rangle\left|0_{x}\right\rangle \\
& =\sqrt{F}\left|\tilde{\psi}_{00}^{x}\right\rangle\left|0_{x}\right\rangle+\sqrt{D}\left|\tilde{\psi}_{01}^{x}\right\rangle\left|1_{x}\right\rangle, \\
|\psi\rangle\left|1_{x}\right\rangle & \rightarrow U|\psi\rangle\left|1_{x}\right\rangle \\
& =\sqrt{D}\left|\tilde{\psi}_{10}^{x}\right\rangle\left|0_{x}\right\rangle+\sqrt{F}\left|\tilde{\psi}_{11}^{x}\right\rangle\left|1_{x}\right\rangle, \\
\left|\tilde{\psi}_{m n}^{x}\right\rangle & \left.\equiv\left\langle m_{x}|U| \psi\right\rangle\left|n_{x}\right\rangle /\left|\left\langle m_{x}|U| \psi\right\rangle\right| n_{x}\right\rangle \mid,
\end{aligned}
$$

for $x=+, \times$ and $m, n=0,1$, where $F+D=1$, and $|\psi\rangle$ is the initial state of Eve's probe and $\left|\tilde{\psi}_{m n}^{x}\right\rangle$ is its normalized state after interaction. The four possible states of $\left|\tilde{\psi}_{m n}^{x}\right\rangle$ are not necessarily orthogonal to each other, but all scalar products other than $\left\langle\tilde{\psi}_{11}^{x} \mid \tilde{\psi}_{00}^{x}\right\rangle=$ $\left\langle\tilde{\psi}_{00}^{x} \mid \tilde{\psi}_{11}^{x}\right\rangle=\left\langle\tilde{\psi}_{10}^{x} \mid \tilde{\psi}_{01}^{x}\right\rangle=\left\langle\tilde{\psi}_{01}^{x} \mid \tilde{\psi}_{10}^{x}\right\rangle \equiv \mathcal{V}$ must be zero and $\mathcal{V}$ must equal $F-D$ in order to symmetrize the strategy [25].

Let us calculate the probabilities that Bob and Eve will correctly infer the state transmitted by Alice when Eve uses this eavesdropping strategy. These probabilities are characterized by the conditional probability $P(j \mid i)$ of obtaining outcome $j$, given that state $\rho_{i}$ was transmitted by Alice. Suppose that Alice transmits either $\rho_{0 x}=\left|0_{x}\right\rangle\left\langle 0_{x}\right|$ or $\rho_{1 x}=\left|1_{x}\right\rangle\left\langle 1_{x}\right|$. Bob's marginal density matrices $\rho_{i x}^{B}$, and Eve's, $\rho_{i x}^{E}$, after the signal-probe interaction and without learning each other's measurement outcomes (nonselective measurement), are easily calculated as

$$
\rho_{0 x}^{B}=\mathcal{E}^{B}\left(\rho_{0 x}\right)=\operatorname{tr}_{E} U|\psi\rangle\langle\psi| \otimes \rho_{0 x} U^{-1}
$$




$$
\begin{aligned}
& =F \rho_{0 x}+D \rho_{1 x}, \\
\rho_{1 x}^{B} & =\mathcal{E}^{B}\left(\rho_{1 x}\right)=\operatorname{tr}_{E} U|\psi\rangle\langle\psi| \otimes \rho_{1 x} U^{-1} \\
& =D \rho_{0 x}+F \rho_{1 x}, \\
\rho_{0 x}^{E} & =\mathcal{E}^{E}\left(\rho_{0 x}\right)=\operatorname{tr}_{B} U|\psi\rangle\langle\psi| \otimes \rho_{0 x} U^{-1} \\
& =F \sigma_{00}^{x}+D \sigma_{01}^{x}, \\
\rho_{1 x}^{E} & =\mathcal{E}^{E}\left(\rho_{1 x}\right)=\operatorname{tr}_{B} U|\psi\rangle\langle\psi| \otimes \rho_{1 x} U^{-1} \\
& =D \sigma_{10}^{x}+F \sigma_{11}^{x},
\end{aligned}
$$

where $\sigma_{m n}^{x}=\left|\tilde{\psi}_{m n}^{x}\right\rangle\left\langle\tilde{\psi}_{m n}^{x}\right|$ and $\mathcal{E}(\rho)$ is a trace-preserving, completely positive, linear map of the density operators of Alice, and Eqs. (2.5)-(2.8) define the unitary representation [26 28] of this map. When Bob performs a standard measurement on the sifted key, the conditional probabilities of Bob's inference of his signal $j$ when Alice sends signal $i$ are, for $x=+, \times$ and $i, j=0,1$,

$$
\begin{aligned}
P_{x}^{A B}(j \mid i) & =\operatorname{tr}\left(\rho_{i x}^{B}\left|j_{x}\right\rangle\left\langle j_{x}\right|\right) \\
& =\left\{\begin{array}{l}
F=\frac{1+\mathcal{V}}{2} \text { if } i=j \\
D=\frac{1-\mathcal{V}}{2} \text { if } i \neq j
\end{array} .\right.
\end{aligned}
$$

On the other hand, Eve's strategy is first to distinguish between two mutually orthogonal sets $S_{i}=\left\{\sigma_{i 0}^{x}, \sigma_{i 1}^{x}\right\}(i=0,1)$ that can be perfectly separated with a standard measurement. She next performs a measurement that distinguishes between $\sigma_{00}^{x}$ and $\sigma_{11}^{x}$ or between $\sigma_{01}^{x}$ and $\sigma_{10}^{x}$, which are not necessary mutually orthogonal $\left(\operatorname{tr} \sigma_{00}^{x} \sigma_{11}^{x}=\operatorname{tr} \sigma_{01}^{x} \sigma_{10}^{x} \neq 0\right)$, that gives the smallest possible error probability. This is the best she can do in terms of the information gained from the sifted key [25]. It is well known that such a measurement is realized by standard measurement in the basis in the Hilbert space spanned by $\left|\tilde{\psi}_{00}^{x}\right\rangle$ and $\left|\tilde{\psi}_{11}^{x}\right\rangle$ or by $\left|\tilde{\psi}_{01}^{x}\right\rangle$ and $\left|\tilde{\psi}_{10}^{x}\right\rangle$ that straddles these vectors [29] 33]. This measurement gives the conditional probabilities of Eve's inference of her signal $j$ when Alice sends signal $i$ as

$$
\begin{aligned}
P_{x}^{A E}(j \mid i) & =\operatorname{tr}\left(\rho_{i x}^{E} \widehat{\Pi}_{j}^{x}\right) \\
& =\left\{\begin{array}{l}
\frac{1}{2}\left(1+\mathcal{D}_{o p t}\right)=\frac{1+\sqrt{1-\mathcal{V}^{2}}}{2} \text { if } i=j \\
\frac{1}{2}\left(1-\mathcal{D}_{\text {opt }}\right)=\frac{1-\sqrt{1-\mathcal{V}^{2}}}{2} \text { if } i \neq j
\end{array},\right.
\end{aligned}
$$

where $\mathcal{D}_{\text {opt }}=\operatorname{tr}\left|\sigma_{00}^{x}-\sigma_{11}^{x}\right|=\operatorname{tr}\left|\sigma_{01}^{x}-\sigma_{10}^{x}\right|=\sqrt{1-\mathcal{V}^{2}}$ is the distance between $\sigma_{00}^{x}$ and $\sigma_{11}^{x}$ and between $\sigma_{01}^{x}$ and $\sigma_{10}^{x}$ in the trace-class norm, and $\widehat{\Pi}_{0}^{x}$ and $\widehat{\Pi}_{1}^{x}$ are the projection-valued measures (PVMs) corresponding to the above detection strategy to distinguish between $\rho_{0 x}^{E}$ and $\rho_{1 x}^{E}$. (Eve also knows when Bob has received an error) [21,33 36. Finally, upon assuming equal a priori probabilities $p_{0+}=p_{1+}=p_{0 \times}=p_{1 \times}$, Bob's average probability ( a posteriori probability) of correct (incorrect) inference of the state transmitted by Alice, $Q_{c}^{B}\left(Q_{e}^{B}\right)$, is given by $\frac{1}{2}\left(P_{+}^{A B}(j \mid i)+P_{\times}^{A B}(j \mid i)\right)$ with $i=j(i \neq j)$ and Eve's average probability, $Q_{c}^{E}\left(Q_{e}^{E}\right)$, is given by $\frac{1}{2}\left(P_{+}^{A E}(j \mid i)+P_{\times}^{A E}(j \mid i)\right)$ with $i=j(i \neq j)$. Thus, $Q_{c}^{B}=\frac{1+\mathcal{V}}{2}$ and $Q_{c}^{E}=\frac{1+\sqrt{1-\mathcal{V}^{2}}}{2}$ gives Bob's and Eve's fidelity, respectively, and $Q_{e}^{B}=\frac{1-\mathcal{V}}{2}$ and $Q_{e}^{E}=\frac{1-\sqrt{1-\mathcal{V}^{2}}}{2}$ gives Bob's 
and Eve's error probability, respectively. $G^{B}=Q_{c}^{B}-Q_{e}^{B}=\mathcal{V}$ and $G^{E}=Q_{c}^{E}-Q_{e}^{E}=\mathcal{D}_{\text {opt }}$ are convenient measures of Bob's and Eve's information gain 21. Since these measures satisfy $\left(G^{B}\right)^{2}+\left(G^{E}\right)^{2}=\mathcal{D}_{o p t}^{2}+\mathcal{V}^{2}=1$, there is a trade-off relation between Bob's and Eve's information gain.

From an information-theoretic point of view, the mutual information $I_{A B}$ between Alice and Bob and $I_{A E}$ between Alice and Eve concerning Alice's message is more appropriate for evaluating Bob's and Eve's knowledge about the sifted key. Mutual information is the measure of information successfully transmitted from input to output. Since Alice and Bob, in general, cannot distinguish between errors caused by eavesdroppers and errors caused by the environment, they have to assume that all errors are due to potential eavesdroppers. As long as Bob's error rate, $Q_{e}^{B}$, is small, the errors can be accepted and corrected by legitimate users. As a result, Eve can obtain some information about the transmitted data. This information leakage to Eve can be eliminated by privacy amplification [6,7] at the cost of reducing the length of the final key. Privacy amplification requires an exact determination of the upper bound of the information leakage to Eve. Thus, the security and robustness of the final key totally depends on this determination. The simple criterion for obtaining a finite length for the secure final key is still an open question, but the inequality $I_{A B}>I_{A E}$ is believed to provide a fairly good criterion; i.e., if the channel noise is such that $I_{A B}<I_{A E}$ for any potential eavesdropper, then Alice and Bob should consider the transmission channel to be unsafe. On the contrary, if $I_{A B}>I_{A E}$, they may still be able to extract a safe but much shorter cryptographic key. Moreover, in a classical context there is, at least in principle, a way for Alice and Bob to exploit any positive difference, $I_{A B}-I_{A E}$, to create a reliably secret string of key bits that has a length of about $I_{A B}-I_{A E}$ [37 39]. It is therefore important to exactly determine the upper bound of the information leakage to Eve from a quantity that Alice and Bob can evaluate.

In the BB84 protocol, $I_{A B}$ can be evaluated directly and $I_{A E}$ can be determined from the error rate $Q_{e}^{B}$ that Alice and Bob can evaluate. With equiprobable signals, they are given by $I_{A B}=1-H\left(Q_{e}^{B}\right)$ and $I_{A E}=1-H\left(Q_{e}^{E}\right)$, where $H(q)=-q \log _{2} q-(1-q) \log _{2}(1-q)$ is the entropy function (in bits) and is a nonlinear function of $q$. Since $\left(Q_{e}^{B}-1 / 2\right)^{2}+$ $\left(Q_{e}^{E}-1 / 2\right)^{2}=\left\{\left(G^{B}\right)^{2}+\left(G^{E}\right)^{2}\right\} / 4=1 / 4, Q_{e}^{B}$ and $Q_{e}^{E}$ are mutually related. The upper plot in Fig. 1 shows $I_{A B}, I_{A E}$ and $I_{A B}+I_{A E}$ plotted against $Q_{e}^{B}$, and the lower plot shows $G^{B}$ and $G^{E}$. From this figure, it is clear that there is a trade-off relation between $I_{A B}$ and $I_{A E}$ as well as a trade-off relation between $G^{B}$ and $G^{E}$. The sum $I_{A B}+I_{A E}$ never exceeds unity $\left(I_{A B}+I_{A E} \leq 1\right)$.

The last inequality, $I_{A B}+I_{A E} \leq 1$, is closely related to the information exclusion principle. This is because the above eavesdropping strategy can be alternatively viewed as a method for simultaneously measuring non-commuting observables. To see this, consider the unitary operation in Eqs. (2.2) and (2.3) with $x=+, F=1(D=0)$. This operation is called measurement of intensity $\gamma$, where $\left\langle\tilde{\psi}_{11}^{+} \mid \tilde{\psi}_{00}^{+}\right\rangle=\left\langle\tilde{\psi}_{00}^{+} \mid \tilde{\psi}_{11}^{+}\right\rangle=\cos \gamma=\mathcal{V}$. 24 When Alice and Bob have chosen the basis +, Eve causes no disturbance and obtains information about the bit to the extent that she can distinguish the two vectors $\left|\tilde{\psi}_{00}^{+}\right\rangle$and $\left|\tilde{\psi}_{11}^{+}\right\rangle$, whose error probability is $\frac{1-\sqrt{1-\mathcal{V}^{2}}}{2}$. Conversely, if Alice and Bob have chosen the basis $\times$, Eve learns nothing and introduces an error with probability $\frac{1-\mathcal{V}}{2}$. Bob's and Eve's information gains when Alice transmits bits with the + basis are therefore $I_{A B}^{+}=1$ and $I_{A E}^{+}=1-H\left(\frac{1-\sqrt{1-\mathcal{V}^{2}}}{2}\right)$, 
and their information gains when Alice transmits the bits with the $\times$ basis are $I_{A B}^{\times}=$ $1-H\left(\frac{1-\mathcal{V}}{2}\right)$ and $I_{A E}^{\times}=0$. Thus, this operation is asymmetric with respect to the basis used in which Eve obtains information on the bits sent with one basis at the cost of a disturbance in the bits sent with the other basis. In this operation, Eve obtains information only about the observable $P_{+}\left(=\left|i_{+}\right\rangle\left\langle i_{+}\right|\right)$of the + basis on the system, while Bob obtains information about both $P_{+}$and $P_{\times}\left(=\left|i_{\times}\right\rangle\left\langle i_{\times}\right|\right)$of the $\times$basis. Thus, when Bob observes $P_{\times}$ and Eve observes $P_{+}$, the above operation provides a method for simultaneously measuring complementary observables, $P_{+}$and $P_{\times}$in which the outcome for Eve gives the information $I_{A E}^{+}=I\left(\rho ; P_{+}\right)$and that for Bob gives $I_{A B}^{\times}=I\left(\rho ; P_{\times}\right)$satisfying $I_{A E}^{+}+I_{A B}^{\times} \leq 1$.

When we extend this argument to the symmetric operation associated with an optimal eavesdropping strategy, we find $I_{A B}^{+}=I_{A B}^{\times} \equiv 1-H\left(\frac{1-\mathcal{V}}{2}\right)$ and $I_{A E}^{+}=I_{A E}^{\times} \equiv 1-H\left(\frac{1-\sqrt{1-\mathcal{V}^{2}}}{2}\right)$ because Bob's and Eve's information gains are independent of the basis Alice chose. We thus find that the symmetric operation provides a method for simultaneously measuring two complementary observables, $P_{+}$and $P_{\times}$, even when Bob observes $P_{+}$. In this case, the outcome for Eve gives the information $I_{A E}^{\times}=I\left(\rho ; P_{\times}\right)$and that for Bob gives $I_{A B}^{+}=I\left(\rho ; P_{+}\right)$. When we also take into account the fact that the sifted key involves only the data for which Alice's and Bob's bases agree, the above arguments imply that Bob's average information gain on the sifted key is given by

$$
I_{A B}=\frac{1}{2}\left\{I\left(\rho_{i \times} ; P_{\times}\right)+I\left(\rho_{i+} ; P_{+}\right)\right\},
$$

whereas Eve's information gain is given by

$$
I_{A E}=\frac{1}{2}\left\{I\left(\rho_{i \times} ; P_{+}\right)+I\left(\rho_{i+} ; P_{\times}\right)\right\}
$$

for the symmetric operation.

We can now see that the information exclusion principle leads to the inequality $I_{A B}+I_{A E} \leq 1$. Since the bases Alice and Bob used in the BB84 protocol are conjugate, $\left|\left\langle 0_{\times} \mid 0_{+}\right\rangle\right|=\left|\left\langle 0_{\times} \mid 1_{+}\right\rangle\right|=\left|\left\langle 1_{\times} \mid 0_{+}\right\rangle\right|=\left|\left\langle 1_{\times} \mid 1_{+}\right\rangle\right|=1 / \sqrt{2}$ holds, and it follows from the information exclusion principle that the inequalities

$$
\begin{aligned}
& I\left(\rho_{i+} ; P_{+}\right)+I\left(\rho_{i+} ; P_{\times}\right) \leq 1 \\
& I\left(\rho_{i \times} ; P_{+}\right)+I\left(\rho_{i \times} ; P_{\times}\right) \leq 1
\end{aligned}
$$

should hold. Equations (2.11) and (2.12) and inequalities (2.13) and (2.14) imply that $I_{A B}+I_{A E} \leq 1$. We therefore conclude that the bound on the sum of Bob's and Eve's information $I_{A B}+I_{A E} \leq 1$ is a direct consequence of the information exclusion principle; that is, the sum can never exceed the maximal amount of information that can be encoded in a two-state system. This condition must be met for the BB84 protocol to be secure. It is therefore essential in the BB84 protocol to limit the size of the signal space $N$, as is easily found from Eq. (2.1). Thus, the conventional BB84 protocol requires use of a single-photon carrier with a limited degree for freedom of information encoding such as polarization encoding. Meeting this condition ensures that no eavesdropping strategy can break this bound. Then, we can safely say that the information leakage to Eve $I_{A E}$ is bounded by $1-I_{A B}$ which Alice and Bob can also evaluate from the bit error rate in Bob's data. Only in such a case, can we establish a provably secure final key by the subsequent privacy amplification. 
It is helpful for later discussion to point out that the information exclusion principle is directly related to the fundamental relation between fringe visibility $\mathcal{V}$ and which-way information (path distinguishability) $\mathcal{D}_{\text {opt }}$ in one-particle interferometry 40 45. To demonstrate this point, we note that the identities $\left|0_{\times}\right\rangle\left\langle 0_{\times}|+| 1_{\times}\right\rangle\left\langle 1_{\times}|=| 0_{+}\right\rangle\left\langle 0_{+}|+| 1_{+}\right\rangle\left\langle 1_{+}\right| \equiv I$, $\left|0_{\times}\right\rangle\left\langle 1_{\times}|+| 1_{\times}\right\rangle\left\langle 0_{\times}|\equiv| 0_{+}\right\rangle\left\langle 0_{+}|-| 1_{+}\right\rangle\left\langle 1_{+}\right|$, and $\left|0_{+}\right\rangle\left\langle 1_{+}|+| 1_{+}\right\rangle\left\langle 0_{+}|\equiv| 0_{\times}\right\rangle\left\langle 0_{\times}|-| 1_{\times}\right\rangle\left\langle 1_{\times}\right|$ hold for a two-state system. We then find that Bob's marginal density matrices $\rho_{0 x}^{B}$ or $\rho_{1 x}^{B}$ can be rewritten in terms of the complementary basis as

$$
\begin{aligned}
\rho_{0 \times}^{B} & =\frac{1}{2}\left\{\left|0_{+}\right\rangle\left\langle 0_{+}|+| 1_{+}\right\rangle\left\langle 1_{+}\right|+\mathcal{V}\left(\left|0_{+}\right\rangle\left\langle 1_{+}|+| 1_{+}\right\rangle\left\langle 0_{+}\right|\right)\right\}, \\
\rho_{1 \times}^{B} & =\frac{1}{2}\left\{\left|0_{+}\right\rangle\left\langle 0_{+}|+| 1_{+}\right\rangle\left\langle 1_{+}\right|-\mathcal{V}\left(\left|0_{+}\right\rangle\left\langle 1_{+}|+| 1_{+}\right\rangle\left\langle 0_{+}\right|\right)\right\}, \\
\rho_{0+}^{B} & =\frac{1}{2}\left\{\left|0_{\times}\right\rangle\left\langle 0_{\times}|+| 1_{\times}\right\rangle\left\langle 1_{\times}\right|+\mathcal{V}\left(\left|0_{\times}\right\rangle\left\langle 1_{\times}|+| 1_{\times}\right\rangle\left\langle 0_{\times}\right|\right)\right\}, \\
\rho_{1+}^{B} & =\frac{1}{2}\left\{\left|0_{\times}\right\rangle\left\langle 0_{\times}|+| 1_{\times}\right\rangle\left\langle 1_{\times}\right|-\mathcal{V}\left(\left|0_{\times}\right\rangle\left\langle 1_{\times}|+| 1_{\times}\right\rangle\left\langle 0_{\times}\right|\right)\right\} .
\end{aligned}
$$

These equations are isomorphic to the equations describing one-particle interferometry where $\mathcal{V}$ gives the fringe visibility and $\mathcal{D}_{\text {opt }}=\sqrt{1-\mathcal{V}^{2}}$ gives the maximal which-way information (path distinguishability), satisfying $\mathcal{D}^{2}+\mathcal{V}^{2} \leq \mathcal{D}_{\text {opt }}^{2}+\mathcal{V}^{2}=1$ [40,41]. Note that the initial states that Alice transmitted are given by setting $\mathcal{V}=1$ in these equations. This implies that the noise introduced by eavesdropping reduces the coherence (the off-diagonal terms) of the initial states, and that Bob's bit error probability $Q_{e}^{B}=\frac{1-\mathcal{V}}{2}$ due to eavesdropping can also be detected by observing the fringe visibility $\mathcal{V}$ in some kinds of interferometry.

To summarize this section, the security of the BB84 protocol totally relies on the quantum mechanical complementarity. This complementarity gives a firm basis for exact determination of the upper bound of the information leakage to eavesdroppers. This enables the security of the final key for the BB84 protocol to be mathematically provable.

\section{BB84 PROTOCOL USING TWO COHERENT STATES AND THEIR}

\section{SUPERPOSITION}

The quantum mechanical complementarity and use of a single-photon carrier ensures that there is an upper bound on the information leakage to eavesdroppers and enables us to determine this bound from the information gain of a legitimate user. The requirement for complementarity to be valid states that the conjugate bases must belong to the same signal space. In other words, if the mutually complementary observables and bases are chosen within the same signal-state space, the BB84 protocol can be constructed. This requirement can of course be satisfied when the polarization space of a single photon is used to encode

information. For this purpose, we require a single-photon source, which has not yet been realized. To overcome this difficulty, a self-checking source, the validity of which can be self-checked, has been devised by Mayers et al. 446,47]

Alternatively, many experimental implementations of BB84 have used weak coherent pulses (WCP), rather than single photons; in these implementations, four equiprobable states given by 


$$
\begin{aligned}
& \left|0_{0}^{w c p}\right\rangle=|\alpha\rangle_{1}|\alpha\rangle_{2}, \quad\left|1_{0}^{w c p}\right\rangle=|\alpha\rangle_{1}|-\alpha\rangle_{2} \\
& \left|0_{\pi / 2}^{w c p}\right\rangle=|\alpha\rangle_{1}|i \alpha\rangle_{2}, \quad\left|1_{\pi / 2}^{w c p}\right\rangle=|\alpha\rangle_{1}|-i \alpha\rangle_{2}
\end{aligned}
$$

were used. 4853 Note that $| \pm i \alpha\rangle=\left(e^{\mp \frac{\pi}{4}} / \sqrt{2}\right)[|\alpha\rangle \mp i|-\alpha\rangle]+O\left(\alpha^{2}\right)$. Therefore, if we consider only the first order in $\alpha$ (i.e., consider only a single-photon component), the four states would behave much like the ideal BB84 states. However, if we consider higher orders in $\alpha$, the two states in one basis $\left|i_{0}^{w c p}\right\rangle$ are no longer linear combinations of the two states in the other basis $\left|i_{\pi / 2}^{w c p}\right\rangle$, and thus do not satisfy the above requirement [54]. As a result, this implementation is vulnerable to eavesdropping. When $\alpha$ is large, these states are four non-orthogonal states lying in a four-dimensional signal state space instead of two sets of two orthogonal states lying in the two-dimensional signal state space used in the original single-photon implementation. There are eavesdropping strategies that make use of the linear independence of the four states. Figure 2 illustrates the relevant subspace of the four states in the entire Hilbert space (the Fock space). Because of the linear independence of the states, there are non-overlapping subspaces in the four states. The states lying in this subspace can be perfectly distinguished from each other, and a skillful eavesdropper can make use of this flaw to obtain information about the key without detection 13 15, 54 .

For example, Reid has described the conclusive-measurement attack, in which Eve can sometimes get full information by using an appropriate "positive operator-valued measure" (POVM) 19, 28, 33,55,56 that conclusively distinguishes such linearly independent states [15]. Such measurement yields no information about the state most of the time, but it sometimes identifies the state unambiguously. Another strategy, called the generalized beamsplitter attack, has also been reported on by several authors [13,48,49, 57]. Since the polarization and photon number are independent observables, there is no problem in principle in selecting a few pulses with two or more photons and separating them into two one-photon pulses without changing the polarization, for example, by means of quantum nondemolition measurement [58]. Both these attacks are fatal, in particular, if the channel loss between Alice and Bob is large enough. This is because Eve can recreate the state near Bob and send it to him whenever she is able to measure the signal state unambiguously and can suppress the signal without causing errors and reducing the bit rate by substituting a less lossy channel. As a result, Eve can obtain information about the key seemingly without introducing errors in the transmission. In addition, most importantly, there is no security proof for the BB84 with WCP implementations as well as the scheme using the two nonorthogonal states [5]. This is because the quantum mechanical complementary can not work effectively in the WCP implementation, and there is no principle for reliably estimating the upper bound of the information leakage to eavesdroppers. This weakness of the WCP scheme arises because the states $\left|i_{0}^{w c p}\right\rangle$ and $\left|i_{\pi / 2}^{w c p}\right\rangle$ are linearly independent if we consider the multi-photon components of the signal states [54]. Thus, the use of the four coherent states in Eq. (3.1) with a large $\alpha$ is inappropriate for the BB84 protocol, and Alice and Bob must use dim coherent pulses each of which, on average, typically contain 0.1 photons for the WCP scheme to approximate the single-photon scheme.

Nevertheless, quantum mechanical complementarity does not forbid us to use the multiphoton state to implement the BB84 protocol. To see this, we consider the scheme depicted in Fig. 囯. Two nearly orthogonal coherent states $|\alpha\rangle$ and $|-\alpha\rangle$ are used to carry the key and 
the superposition of these states $(|\alpha\rangle \pm|-\alpha\rangle) / \sqrt{2(1 \pm \kappa)}$ is used to prevent eavesdropping. Here, $\kappa$ is the overlap of the two coherent states $|\alpha\rangle$ and $|-\alpha\rangle$; i.e., $\kappa=|\langle\alpha \mid-\alpha\rangle|=e^{-2|\alpha|^{2}}$. These states are the "Schrödinger's cat states" and are parity eigenstates that lie within the relevant two-dimensional signal subspace spanned by $\{|\alpha\rangle,|-\alpha\rangle\}$ in the Fock space [59 61]. These four states satisfy the requirement for quantum mechanical complementarity to be valid, and would therefore behave much like ideal BB84 states.

In the following, we describe the protocol and explain how eavesdropping is detected. Consider the following protocol using only three, instead of four, states. This protocol is not the original BB84 protocol and is less efficient, but it is enough to explain the basic idea of the present scheme.

1. Alice first chooses a subset of random positions within a sequence of data being transmitted.

2. She then transmits random bits encoded with a set of nearly orthogonal states $\left|0_{+}\right\rangle=$ $|\alpha\rangle$ and $\left|1_{+}\right\rangle=|-\alpha\rangle$ for the chosen subset (the first subset) which provides a raw key.

3. She also transmits either $\left|0_{\times}\right\rangle=(|\alpha\rangle-|-\alpha\rangle) / \sqrt{2(1-\kappa)}$ or $\left|1_{\times}\right\rangle=(|\alpha\rangle+$ $|-\alpha\rangle) / \sqrt{2(1+\kappa)}$ for the remaining subset (the second subset) which will be used only to detect eavesdropping.

4. Alice also transmits a strong local oscillator beam (LO) with its polarization rotated so as to be orthogonal to the signal beam on the same channel by mixing the beams on a polarizing beamsplitter. The mixed beams are then transmitted to Bob.

5. Bob uses a polarizing beamsplitter to separate the LO from the channel. The polarization of the LO is rotated by $\pi / 2$ using a $\lambda / 2$-plate so as to match that of the signal. With this strong LO, Bob performs balanced homodyne detection 62 to measure the single field-quadrature $\hat{X}(\theta)=\hat{x}_{a} \cos \theta+\hat{p}_{a} \sin \theta=(1 / \sqrt{2})\left[e^{-i \theta} \hat{a}+e^{i \theta} \hat{a}^{\dagger}\right]$ of the signal when he receives it, where $\hat{x}_{a}=(1 / \sqrt{2})\left[\hat{a}+\hat{a}^{\dagger}\right], \hat{p}_{a}=(1 / \sqrt{2} i)\left[\hat{a}-\hat{a}^{\dagger}\right]$. If we assume that $\alpha$ is real for simplicity, then $\theta$ is the advance of the signal phase relative to the LO phase (which is Bob's controllable parameter). He randomly varies $\theta$ between 0 and $\pi / 2$ by changing the LO phase with phase shifter $\mathrm{A}$. (It is possible for Alice and Bob to calibrate the phase $\theta$ without introducing any vulnerability.)

6. After transmission, Alice publicly announces the positions of the first and second data subsets. Alice and Bob then discard the part of the first subset of data for which Bob measured $\hat{p}_{a}(\theta=\pi / 2)$ and the part of the second subset of data for which he measured $\hat{x}_{a}(\theta=0)$. Bob can obtain the sifted key from the first subset of the remaining data.

For the moment, let us consider a perfect detector with unit efficiency and negligible channel loss. The effects of the detection efficiency and channel loss are considered in the next section. In terms of the sifted key, the conditional probability distributions $p_{i+}\left(x_{a}\right)$ of Bob's output $x$ when Alice transmits signal $i$ obey the Gaussian distributions:

$$
\begin{aligned}
p_{0+}\left(x_{a}\right) & =\operatorname{Tr}\left|0_{+}\right\rangle\left\langle 0_{+} \mid x_{a}\right\rangle\left\langle x_{a}\right| \\
& =\frac{1}{\pi^{1 / 2}} \exp \left[-\left(x_{a}-\langle\alpha\rangle\right)^{2}\right],
\end{aligned}
$$




$$
\begin{aligned}
p_{1+}\left(x_{a}\right) & =\operatorname{Tr}\left|1_{+}\right\rangle\left\langle 1_{+} \mid x_{a}\right\rangle\left\langle x_{a}\right| \\
& =\frac{1}{\pi^{1 / 2}} \exp \left[-\left(x_{a}+\langle\alpha\rangle\right)^{2}\right],
\end{aligned}
$$

where $\langle\alpha\rangle=\sqrt{2}|\alpha|$. The standard strategy for Bob to correctly infer the state transmitted by Alice is to set the decision threshold at $x_{a}=0$; i.e., he sets the bit value to 0 when he obtains $x_{a} \geq 0$ and to 1 when he obtains $x_{a}<0$. Then, his average error probability has finite value $Q_{e}^{B}(\alpha)=\frac{1}{2} \operatorname{Erfc}[\langle\alpha\rangle]$, where $\operatorname{Erfc}[x]$ is the complementary error function defined by $\operatorname{Erfc}[x] \equiv(1 / \sqrt{2 \pi}) \int_{x}^{\infty} \exp \left[-\tau^{2}\right] d \tau[63]$. This is because the two coherent states $|\alpha\rangle$ and $|-\alpha\rangle$ are not orthogonal. Bob also checks the second subset of remaining data to detect possible eavesdropping. Provided that Alice transmits the $\left|1_{\times}\right\rangle$state for the second subset, the associated conditional probability distribution $p_{1 \times}\left(p_{a}\right)$ is

$$
p_{1 \times}\left(p_{a}\right)=\operatorname{Tr}\left|1_{\times}\right\rangle\left\langle 1_{\times} \mid p_{a}\right\rangle\left\langle p_{a}\right|=\frac{1}{(1+\kappa)} \frac{1}{\pi^{1 / 2}} \exp \left[-p_{a}^{2}\right]\left\{1+\sin \left[2\langle\alpha\rangle p_{a}\right]\right\} .
$$

Therefore, when Bob builds up the probability distribution $p_{1 \times}\left(p_{a}\right)$ of getting outcome $p_{a}$ upon measurement of $\hat{p}_{a}$, the distribution should have interference fringes with a period of $\pi /\langle\alpha\rangle$ in the absence of eavesdropping [59,64.

To eavesdrop, Eve can, in principle, use a symmetric strategy by applying a joint unitary operation similar to the one shown in Eqs. (2.2) and (2.3). It must involve complex multiphoton interaction between the single-mode field of the signal states and the probe system, and a physical mechanism that would enable such an operation has been unknown. Even if such an operation is realized, we can safely conclude that our proposed scheme is as secure as the single-photon case as far as this strategy is concerned by an argument similar to the single-photon case. This conclusion is closely related to the fact that the quantum mechanical superposition of macroscopically distinguishable states cannot be noninvasively measured 65.66, which is essentially a direct consequence of the quantum mechanical complementarity. Moreover, this scheme is secure against a conclusive-measurement attack because the two mutually conjugate sets $\left|i_{+}\right\rangle$and $\left|i_{\times}\right\rangle$are linearly dependent. In the rest of the paper, we thus consider only a simple strategy that can only be used for cryptographic schemes using multi-photon states, that is, a beamsplitter attack. We show that the intentional eavesdropping activity will be detected by the legitimate users, and explain how the eavesdropping is detected.

We consider the following scenario. Eve uses a beam splitter (BS) to sample part of the signal. She sends Bob the part of the signal transmitted through the BS and measures the reflected part to gain information about the signal. What we want to know is how much she can learn and how much she disturbs the signal state. For this purpose, it is sufficient to calculate Eve's error rate $Q_{e}^{E}$ on the sifted key for this particular scheme. If we denote the signal mode defined by the quantum channel as $a$ and an auxiliary mode introduced at the $\mathrm{BS}$ as $b$, the associated joint unitary operation of the BS on coherent state input is

$$
\begin{aligned}
& \left|0_{+}\right\rangle_{a}|0\rangle_{b} \rightarrow U_{B S}|\alpha\rangle_{a}|0\rangle_{b}=|\sqrt{T} \alpha\rangle_{a}|-\sqrt{R} \alpha\rangle_{b}, \\
& \left|1_{+}\right\rangle_{a}|0\rangle_{b} \rightarrow U_{B S}|-\alpha\rangle_{a}|0\rangle_{b}=|-\sqrt{T} \alpha\rangle_{a}|\sqrt{R} \alpha\rangle_{b},
\end{aligned}
$$

where $T=\sqrt{1-R^{2}}$ is the transmission coefficient of the BS 667. On the other hand, the same unitary operation transforms the $\left|1_{\times}\right\rangle$state as 


$$
\left|0_{\times}\right\rangle_{a}|0\rangle_{b} \rightarrow U_{B S} \frac{|\alpha\rangle_{a}+|-\alpha\rangle_{a}}{\sqrt{2(1+\kappa)}}|0\rangle_{b}=\frac{1}{\sqrt{2(1+\kappa)}}\left\{|\sqrt{T} \alpha\rangle_{a}|-\sqrt{R} \alpha\rangle_{b}+|-\sqrt{T} \alpha\rangle_{a}|\sqrt{R} \alpha\rangle_{b}\right\} .
$$

This indicates that the resultant state is entangled with respect to modes $a$ and $b$ even though the BS is a linear device. Therefore, noise is inevitably introduced into the transmission of the $\left|1_{\times}\right\rangle$state. The associated marginal density matrices, $\rho_{i+}^{B}$ and $\rho_{1 \times}^{B}$ for Bob and $\rho_{i+}^{E}$ and $\rho_{1 \times}^{E}$ for Eve after the beamsplitter are calculated as

$$
\begin{aligned}
\rho_{i+}^{B}= & \operatorname{tr}_{E} U_{B S} \rho_{i+}^{a} \otimes|0\rangle_{b}\left\langle 0\left|U_{B S}^{-1}=\right| \pm \sqrt{T} \alpha\right\rangle_{a}\langle \pm \sqrt{T} \alpha|, \\
\rho_{1 \times}^{B}= & \operatorname{tr}_{E} U_{B S} \rho_{1 \times}^{a} \otimes|0\rangle_{b}\langle 0| U_{B S}^{-1} \\
= & \frac{1}{2(1+\kappa)}\left\{|\sqrt{T} \alpha\rangle_{a}\langle\sqrt{T} \alpha|+|-\sqrt{T} \alpha\rangle_{a}\langle-\sqrt{T} \alpha|\right. \\
& \left.\quad+\mathcal{V}_{B}\left(|\sqrt{T} \alpha\rangle_{a}\langle-\sqrt{T} \alpha|+|-\sqrt{T} \alpha\rangle_{a}\langle\sqrt{T} \alpha|\right)\right\}, \\
\rho_{i+}^{E}= & \operatorname{tr}_{B} U_{B S} \rho_{i+}^{a} \otimes|0\rangle_{b}\left\langle 0\left|U_{B S}^{-1}=\right| \mp \sqrt{R} \alpha\right\rangle_{a}\langle\mp \sqrt{R} \alpha|, \\
\rho_{1 \times}^{E}= & \operatorname{tr}_{B} U_{B S} \rho_{1 \times}^{a} \otimes|0\rangle_{b}\langle 0| U_{B S}^{-1} \\
= & \frac{1}{2(1+\kappa)}\left\{|\sqrt{R} \alpha\rangle_{a}\langle\sqrt{R} \alpha|+|-\sqrt{R} \alpha\rangle_{a}\langle-\sqrt{R} \alpha|\right. \\
& \left.+\mathcal{V}_{E}\left(|\sqrt{R} \alpha\rangle_{a}\langle-\sqrt{R} \alpha|+|-\sqrt{R} \alpha\rangle_{a}\langle\sqrt{R} \alpha|\right)\right\},
\end{aligned}
$$

where $\rho_{i+}^{a}=\left|i_{+}\right\rangle_{a}\left\langle i_{+}|=| \pm \alpha\right\rangle_{a}\left\langle \pm \alpha\left|, \quad \rho_{1 \times}^{a}=\right| 1_{\times}\right\rangle_{a}\left\langle 1_{\times}\left|, \quad \mathcal{V}_{B}=\right|\langle\sqrt{R} \alpha \mid-\sqrt{R} \alpha\rangle\right|=$ $e^{-2(1-T)|\alpha|^{2}}, \mathcal{V}_{E}=|\langle\sqrt{T} \alpha \mid-\sqrt{T} \alpha\rangle|=e^{-2 T|\alpha|^{2}}$ (note that $\left.\mathcal{V}_{B} \mathcal{V}_{E}=\kappa\right)$, and the upper sign (resp. lower sign) corresponds to $i=1$ (resp. $i=0$ ). Provided that Eve uses an optimum decision strategy that results in the smallest possible error when distinguishing two non-orthogonal coherent states $|\sqrt{R} \alpha\rangle_{a}$ and $|-\sqrt{R} \alpha\rangle_{a}$, her error rate $Q_{e}^{E}$ is given by

$$
Q_{e}^{E}=\frac{1-\sqrt{1-\mathcal{V}_{B}^{2}}}{2}
$$

Such an optimum decision strategy can, in principle, be realized [68 70].

What Alice and Bob want to do is to evaluate $Q_{e}^{E}$ or Eve's average information gain $I_{A E}=1-H\left(Q_{e}^{E}\right)$ as a function of disturbance observable in the signal that Bob recorded. When we note that Eq. (3.8) is formally isomorphic to Eq. (2.16), we find that the most appropriate measure of the disturbance is the fringe visibility observable in the probability distribution $p_{1 \times}\left(p_{a}\right)$. From Eq. (3.8), $p_{1 \times}\left(p_{a}\right)$ in the presence of eavesdropping can be easily calculated as

$$
p_{1 \times}\left(p_{a}\right)=\operatorname{Tr} \rho_{1 \times}^{B}\left|p_{a}\right\rangle\left\langle p_{a}\right|=\frac{1}{(1+\kappa)} \frac{1}{\pi^{1 / 2}} \exp \left[-p_{a}^{2}\right]\left\{1+\mathcal{V}_{B} \sin \left[2 \sqrt{T}\langle\alpha\rangle p_{a}\right]\right\}
$$

The fringe visibility is therefore given by $\mathcal{V}_{B}$. Figure 4 shows Eve's average information gain $I_{A E}=1-H\left(Q_{e}^{E}\right)$ calculated from Eq. (3.11) as a function of the fringe visibility $\mathcal{V}_{B}$. 
This figure clearly indicates that the amount of information leakage to eavesdroppers can be estimated from the visibility of the interference fringe in the probability distribution $p_{1 \times}\left(p_{a}\right)$ of getting outcome $p_{a}$ upon homodyne-detection measurement of $\hat{p}_{a}$. It is immediately confirmed that the sum of the squared measures of disturbance $\mathcal{V}_{B}$ and distinguishability $\mathcal{D}_{B}=1-2 Q_{e}^{E}=\sqrt{1-\mathcal{V}_{B}^{2}}$ reaches its expected upper bound of unity; $\mathcal{D}_{B}^{2}+\mathcal{V}_{B}^{2}=1$. This indicates that the information leakage to eavesdroppers for a beamsplitter attack reaches its upper bound as does that for the more sophisticated symmetric eavesdropping strategy. This scheme is thus secure even against the beamsplitter attack even though the multi-photon states are used as a signal carrier.

On the other hand, Bob's information gain $I_{A B}=1-H\left(Q_{e}^{B}\right)$ is easily evaluated by publicly revealing a part of his sifted keys. In the case of single-photon implementation, $I_{A B}$ is expected to be unity for this type of asymmetric attack. Figure 4 compares Bob's information gain $I_{A B}=1-H\left(Q_{e}^{B}\right)$ on the sifted key in the presence of eavesdroppers for the average photon number $|\alpha|^{2}=1$ and 2 under the assumption that he performed homodyne detection followed by the standard decision strategy. In contrast to the singlephoton implementation, Bob's information vanishes in the low fringe-visibility region. This is because the beamsplitter directs the signal light to Eve and the intensity of the signal going to Bob falls to zero.

Figure 4 indicates that to learn about Alice's state with some degree of accuracy, Bob's visibility $\mathcal{V}_{B}$ must not be too large, which implies that the reflection coefficient $1-T$ must not be too small. The requirements for a large information gain and little disturbance are thus incompatible. A large information gain requires a small transmission coefficient, while a small disturbance requires a transmission coefficient close to one, and there is no overlap in the permitted ranges. Therefore, with this QKD scheme, Eve cannot use beamsplitter attack and diverts enough light to gain any useful information without producing a detectable disturbance. This confirms the impossibility of noninvasive measurement of the quantummechanical superposition of macroscopically distinguishable states. The problem for Eve is the vacuum noise from the vacant port of the BS. If she samples only a small part of the signal, to minimize the disturbance, the noise from the vacuum state obscures the information carried by the signal state [14].

\section{DISCUSSION}

The previous section discussed the ideal situation in which channel loss and detector inefficiency can be ignored. In this case, the present scheme enables us to exactly determine the upper bound of the information leakage to eavesdroppers $I_{A E}$ from the fringe visibility of the probability distribution $p_{1 \times}\left(p_{a}\right)$. As a result, its security will, in principle, be provable in the ideal case under the assumption that Alice does send cat states. This would be the principal advantage of the scheme over the conventional WCP scheme. This scheme also offers advantages. First, it involves only quadrature phase measurements, which can be done more efficiently than photon counting. Second, this scheme can use a more intense pulse. These advantages may allow us to improve the transmission efficiency compared to that of the conventional WCP scheme.

However, in the presence of channel loss and detection inefficiency, the above results need to be reconsidered. It is known that the cat state is so fragile that the loss of a single 
photon may easily destroy the interference fringe observed in the probability distribution $p_{1 \times}\left(p_{a}\right)$. Moreover, the decoherence rate of the cat state is proportional to the distance between the two distinguishable coherent states; i.e., it is proportional to $\sqrt{1-\kappa^{2}} \sim|\alpha|$ [59:64]. A cat state with a very large average photon number $|\alpha|^{2}$ decoheres rapidly, and thus the present scheme will not be practical. We here briefly describe the effects of channel loss and detection inefficiency on the present scheme. Channel loss and detection inefficiency are, as usual, modeled by a beam splitter that mixes the signal mode with a vacuum field in an auxiliary mode. Thus, these effects are essentially analogous to the beamsplitter attack. In other words, neither of these effects can be distinguished from eavesdropping by means of local measurement by legitimate users. This would be a weak point in common with the cryptographic scheme using a multi-photon nonclassical state as a signal carrier. Let us assume that the overall channel loss is $\zeta$ and the detector efficiency is $\eta$. The above model then reveals that Eq. (3.12) is correct if we replace $T \rightarrow \varepsilon \eta T$ in $\mathcal{V}_{B}$ and Eq. (3.12). This means that $\mathcal{V}_{B}$ is reduced by a factor $e^{-2(1-\varepsilon \eta) T|\alpha|^{2}}$ and $0 \leq \mathcal{V}_{B} \leq e^{-2(1-\varepsilon \eta)|\alpha|^{2}}$. Thus, unless $\varepsilon \eta \neq 1$, the visibility is less than unity even if $T=1$; i.e., no eavesdropper is present. The legitimate users can measure the detection efficiency locally though. Thus, its effect may be subtracted when determining the upper bound of the information leakage to Eve from the observed visibility. Moreover, if legitimate users have a reliable way to evaluate the channel loss, its effect may also be subtracted. However, when $2(1-\varepsilon \eta)|\alpha|^{2} \gg 0, \mathcal{V}_{B} \approx 0$. Eavesdropping would then be very difficult to detect from the subtle changes in $\mathcal{V}_{B}$, and this scheme would not be practical. Thus, $1-\varepsilon \eta$ and $|\alpha|^{2}$ need to be very small for this scheme to work. In practice, even a minor 3 - $\mathrm{dB}$ loss $(\varepsilon \eta=0.5)$ will make it hard to use this scheme, unless $|\alpha|^{2}$ is small. It is this extreme sensitivity of the nonclassical field state to the environment that enables us to detect eavesdropping. In contrast, the channel loss is simply discarded in the WCP scheme, but this discarding also makes the WCP scheme vulnerable because eavesdroppers have chance to use it while substituting a superior channel to escape detection.

On the other hand, there is a lower bound on $|\alpha|^{2}$ that enables use of the cat state to detect eavesdropping. To evaluate the fringe visibility, there should be at least one oscillation in the distribution $p_{1 \times}\left(p_{a}\right)$ within the Gaussian contour exp $\left[-p_{a}^{2}\right]$. This requirement should impose the inequality $\Delta p_{a}=\pi /(2 \sqrt{2 \varepsilon \eta T}|\alpha|)<2 \sqrt{\ln 2}$. If we note that $\varepsilon \eta T \leq 1,|\alpha|>$ $\pi /(4 \sqrt{2 \ln 2}) \sim 0.67$ is required. Thus, a cat state with an average photon number of the order of unity is appropriate for our scheme. In this sense, what is needed is not a "macroscopic" quantum superposition but a "mesoscopic" quantum superposition which should be easier to create. The present scheme is effective only if a good channel with low loss, a highly efficient detector, and a mesoscopic cat state are available.

Hillery's idea of using phase-sensitive amplifiers to boost the signal and partially compensate for the effect of losses for a cryptographic scheme using the squeezed state is interesting. When a device that can amplify the cat state becomes available in the future, his idea can also be applied to the present scheme: if such a device is used in the secure station in the channel, it can partially compensate for the effect of losses by amplifying only the cat state in accord with the state Alice sends, and otherwise it can compensate the effect by randomly amplifying the signal and discarding a part of the data. [14]

The problem with the cryptographic scheme using a multi-photon nonclassical state comes from the fact that the state after eavesdropping and the state after losses are indistinguishable. A cryptographic scheme using a single photon seems not to have such a problem. 
This is because the state after photon loss is a vacuum state that spans a subspace different from the signal space of a single-photon state. Such a scheme can conclusively distinguish a photon-loss event and uses only the photons that did not get absorbed. Thus, it can overcome $10 \mathrm{~dB}$ of losses. That, in essence, is why conventional quantum cryptography using single photons can work so well in the presence of channel loss and detection inefficiency. We should, however, remember that this is true only with the assumption that the apparatus used by Alice to produce the photon is perfect [9]. To confirm that this assumption is valid, we must check that the signal space is limited to two-dimensional space; i.e., whether the photon source is a good single-photon source. This check can be accomplished through observation of the nonlocal properties inherent in quantum mechanics, such as violation of Bell's inequality [46] and Bell's theorem without inequalities associated with an entangled tripartite system, [71, [7] to ensure that the photon source is a perfect single-photon source. This is because no local photon source other than a perfect single-photon source can reproduce the results observed nonlocally between legitimate users. [46,47] However, note that the rigorous test for a violation of Bell's inequality and Bell's theorem associated with an entangled tripartite system is possible only if the product of the channel loss and detection efficiency is higher than some lower limit of around $80 \%$. [72 74 If the loss and efficiency product is below this lower limit, an auxiliary assumption (a fair-sampling assumption) that the fraction of detected pairs is representative of the entire ensemble is required to rule out any local realistic model that can reproduce the observed results and to prove the security of the BB84 protocol without any loophole. 7274

The current feasibility of the present scheme is limited by the difficulty of preparing the cat state with today's technology in addition to channel loss and detector inefficiency. However, a development of a quantum gate will help us to obtain the cat state through a swapping operation [75] between a coherent state and a more easily created superposition state of a single quanta [60,86].

\section{CONCLUSION}

It is a distinct feature of the ideal BB84 protocol that its security is mathematically provable. This is possible because quantum mechanical complementarity enables us to determine the upper bound of the information leakage to eavesdroppers. We have shown that complementarity allows also use of multi-photon states as a signal carrier in the BB84 QKD protocol, and have described a scheme that uses two nearly orthogonal coherent states to carry the key where the superposition of these states protects the communication channel from eavesdropping. This scheme is based on complementarity as is the conventional BB84 scheme. We expect this scheme to be as secure as the conventional single-photon scheme and secure against any eavesdropping strategy. The disappearance of interference fringes in the homodyne detection used to decode the key clearly indicates eavesdropping activity, and the upper bound of information leakage to eavesdroppers can be exactly determined from the visibility of the interference fringes which is measurable from the homodyne detection. As a result, this scheme will be provably secure as long as a good channel with negligible loss, a highly efficient detector, and a mesoscopic cat state are available.

Unfortunately, this scheme is very sensitive to losses and is not practical in the presence of high channel loss and detection inefficiency. In this case, a single-photon implementation 
would be preferable, although the provable security still requires low channel loss and high detection efficiency.

\section{ACKNOWLEDGMENTS}

We thank Dominic Mayers for his helpful discussions concerning the validity of the present scheme. 


\section{REFERENCES}

[1] S. Wiesner, Sigact News, 15, 78 (1983).

[2] C. H. Bennett, G. Brassard, Proc. of IEEE International Conference on Computers, Systems, and Signal Processing, Bangalore, India, December 1984, pp. 175-179.

[3] A. K. Ekert, Phys. Rev. Lett. 67, 661 (1991).

[4] C. H. Bennett, G. Brassard, and N. D. Mermin, Phys. Rev. Lett. 68, 557 (1992).

[5] C. H. Bennett, Phys. Rev. Lett. 68, 3121 (1992).

[6] C. H. Bennet, G. Brassard, C. Crépeau, and M. Maurer: Generalized privacy amplification. IEEE trans. Inf. Theo. 41, 1915 (1995).

[7] C. H. Bennet, F. Bessette, G. Brassard, L Salvail, and J. Smolin: J. Cryptology 5, 2 (1992).

[8] G. S. Vernam, J. Am. Inst. Electr. Eng. 45, 109 (1926).

[9] D. Mayers, e-print quant-ph/9802025.

[10] B. Huttner and A. K. Ekart, J. Mod. Opt. 41, 2455-2466 (1994).

[11] S. L. Braunstein and H. J. Kimble, Phys. Rev. Lett. 80, 869 (1998).

[12] A. Furusawa, J. L. Sorensen, S. L. Braunstein, C. A. Fuchs, H. J. Kimble, and E. S. Polzik, Science 282, 706 (1998).

[13] T. Ralph, Phys. Rev. A 61, 010303 (2000).

[14] M. Hillery, Phys. Rev. A 61, 022309 (2000).

[15] M. D. Reid, e-print quant-ph/9909030.

[16] M. J. Hall, Phys. Rev. Lett. 74, 3307 (1995).

[17] K. Kraus, Phys. Rev. D 35, 3070 (1987).

[18] W. K. Wootters and B. D. Field, Ann. Phys. NY 191, 363 (1989).

[19] A. Peres, Quantum theory: concepts and methods (Kluwer, Dordrecht, 1998), Chap. 9.

[20] C. M. Caves and P. D. Drummond, Rev. Mod. Phys. 66, 481 (1994).

[21] C. A. Fuchs, N. Gisin, R. B. Griffiths, C-S. Niu, and A. Peres, Phys. Rev. A 56, 1163 (1997).

[22] V. Buzĕk and M. Hillery, Phys. Rev. A 54, 1844 (1996).

[23] D. Bruß, D. P. Divicenzo, A. Ekart, D. A. Fuchs, C. Macchiavello, and J. A. Smolin, Phys. Rev. A 57, 2368 (1998).

[24] N. Gisin and B. Huttner, Phys. Lett. A 228, 13-21 (1997).

[25] J. I. Cirac and N. Gisin, Phys. Lett. A 229, 1-7 (1997). 
[26] B. Schumacher, Phys. Rev. A 54, 2614 (1996).

[27] B. Schumacher and M. A. Nielsen, Phys. Rev. A 54, 2629 (1996).

[28] J. Preskill, Quantum information and computation, Lecture notes for physics 229 in CALTEC (1998), available at http://www.theory.caltech.edu/people/preskill/ph229/.

[29] R. S. Kennedy, Mass. Inst. Tech. Res. Lab. Electron. Quart. Prog. Rep. No. 110, 142 (1974).

[30] E. B. Davis, IEEE Trans. Inf. Theory IT-24, 596 (1978).

[31] B. Huttner, A. Muller, J. D. Gautier, H. Zbinden, and N. Gisin, Phys. Rev. A 54, 3783 (1996).

[32] L. B. Levitin, in Workshop on Physics and Computation: Phys. Comp '92, edited by D. Matzke (IEEE Computer Society Press, Los Alamitos, CA, 1993).

[33] C. W. Helstrom, Quantum detection and estimation theory (Academic, New York, 1976).

[34] L. B. Levitin, in Information Complexity and Control in Quantum Physics, edited by A. Blaquiere, S. Diner, and G. Lochak (Springer, Berlin, 1987), p. 15.

[35] P. Busch, e-print quant-ph/9604014.

[36] G. Jaeger and A. Shimony, Phys. Lett. A 128, 19 (1995).

[37] A. D. Wyner, Bell Syst. Tech. J. 54, 1355 (1975).

[38] I. Csiszár and J. Körner, IEEE Trans. Inf. Theory 24, 339 (1978).

[39] U. M. Maurer, IEEE Trans. Inf. Theory 39, 722 (1992).

[40] B-G. Englert, Phys. Rev. Lett. 77, 2154 (1996).

[41] L. Mandel, Opt. Lett. 16, 1882 (1991).

[42] S. Dürr, T. Nonn, and G. Rempe, Phys. Rev. Lett. 81, 5705 (1998).

[43] G. Jaeger, M. A. Horne, and A. Shimony, Phys. Rev. A 48, 1023 (1993).

[44] G. Jaeger, A. Shimony, and L. Vaidman, Phys. Rev. A 51, 54 (1995).

[45] W. K. Wootters and W. H. Zurek, Phys. Rev. D 19, 473 (1979).

[46] D. Mayers and Andrew Yao, in Proceedings of 39th Annual Symposium on Foundations of Computer Science, 503 (1998), e-print quant-ph/9809039.

[47] D. Mayers, Y. Kohno, Y. Nambu, and A. Tomita, to be published in Phys. Rev. Lett.

[48] H. Yuen, Quantum Semiclass. Opt. 8, 939-949 (1996).

[49] B. Huttner, N. Imoto, N. Gisin, and T. Mor, Phys. Rev. A 51, 1863 (1995).

[50] Y. Mu, J. Seberry, and Y. Zheng, Opt. Commun. 123, 344 (1996).

[51] P. D. Townsend, J. G. Rality, and P. R. Tapster, Electron. Lett. 29, 634 (1993). 
[52] P. D. Townsend, J. G. Rality, and P. R. Tapster, Electron. Lett. 29, 1291 (1993).

[53] P. D. Townsend, Electron. Lett. 30, 809 (1994).

[54] G. Brassard, Tal Mor, and B. C. Sanders, e-print quant-ph/9906074.

[55] E. B. Davies and J. T. Lewis, Comm. Math. Phys. 17, 239 (1970).

[56] K. Kraus, States, Effects, and Operations: Fundamental Notions of Quantum Theory (Springer-Verlag, Berlin, 1983)

[57] H. K. Lo and H. F. Chu, Science 283, 2050 (1999); with supplementary material available at http://www.sciencemag.org/feature/data/984035.sh1.

[58] Y. Yamamoto, in Encyclopedia of Applied Physics, vol. 15, pp. 307-337 (VCH Publishers, 1996).

[59] B. Yurke and D. Stoler, Phys. Rev. Lett. 57, 13 (1986).

[60] P. T. Cochrane, G. J. Milburn, and W. J. Munro, Phys. Rev. A 59, 2631 (1999).

[61] V. Buzek and P. L. Knight, Progress in Optics XXXIV, edited by E. Wolf (NorthHolland, Amsterdam, 1995).

[62] H. P. Yuen and V. W. S. Chen, Optt. Lett. 8, 177 (1983).

[63] M. Osaki, M. Ban, and O. Hirota, Phys. Rev. A 54, 1691 (1996).

[64] D. F. Walls and G. J. Milburn, Phys. Rev. A 31, 2403 (1985).

[65] L. E. Ballentine, Phys. Rev. Lett. 59, 1493 (1987).

[66] A. Peres, Phys. Rev. Lett. 61, 2019 (1988).

[67] S. M. Barnett and P. M. Radmore, Methods in theoretical quantum optics (Clarendon, Oxford, 1997).

[68] M. Sasaki and O. Hirota, Phys. Lett. A 210, 21 (1996).

[69] M. Sasaki, T. S. Usuda, O. Hirota, and A. S. Holevo, Phys. Rev. A 53, 1273 (1996).

[70] M. Sasaki and O. Hirota, Phys. Rev. A 54, 2728 (1996).

[71] D. M. Greenberger, M. A. Horne, A. Shimony, and A. Zeilinger, Am. J. Phys. 58, 731 (1990); 58, 1131 (1990).

[72] J. F. Clauser and M. A. Horne, Phys. Rev. D 10, 526 (1974).

[73] A. Garg and N. D. Mermin, Phys. Rev. D 35, 3831 (1987).

[74] J-A. Larsson, Phys. Rev. A 57, 3145 (1998); A 57, 3304 (1998).

[75] Y. Aharonov and L. Vaidman, e-print quant-ph/9909072.

[76] C. C. Gerry, Phys. Rev. A 59, 4095 (1999). 


\section{FIGURES}

FIG. 1. Upper plot: Bob's information gain $I_{A B}$, Eve's information gain $I_{A E}$ and their sum $I_{A B}+I_{A E}$ are plotted against Bob's error probability $Q_{e}^{B}$ when Eve applys an optimum eavesdropping strategy. Lower plot: measures of information gained by Bob $\left(G^{B}\right)$ and by Eve $\left(G^{E}\right)$ are plotted.

FIG. 2. The relevant subspace of the four weak coherent states in the entire Hilbert space (the Fock space). The parts of the four circles that do not overlap indicate the linear independence of the states.

FIG. 3. The basic idea of the proposed QKD scheme. Alice and Bob use two nearly orthogonal coherent states to carry the key and the superposition of these states (cat states) to protect from eavesdropping. Eavesdropping is detected from the disappearance of the interferential fringes in the distribution of the outcome when a certain quadrature component is measured by the homodyne detection.

FIG. 4. Information leakage to $I_{A E}$ (a solid line) and Bob's average information gain $I_{A B}$ (broken lines) as a function of the fringe visibility $\mathcal{V}_{B}=e^{-2(1-T)|\alpha|^{2}}$ in the probability distribution

$p_{1 \times}\left(p_{a}\right)$ recorded by Bob. $I_{A B}$ was evaluated at the average photon number $|\alpha|^{2}=2$ and $|\alpha|^{2}=1$. 


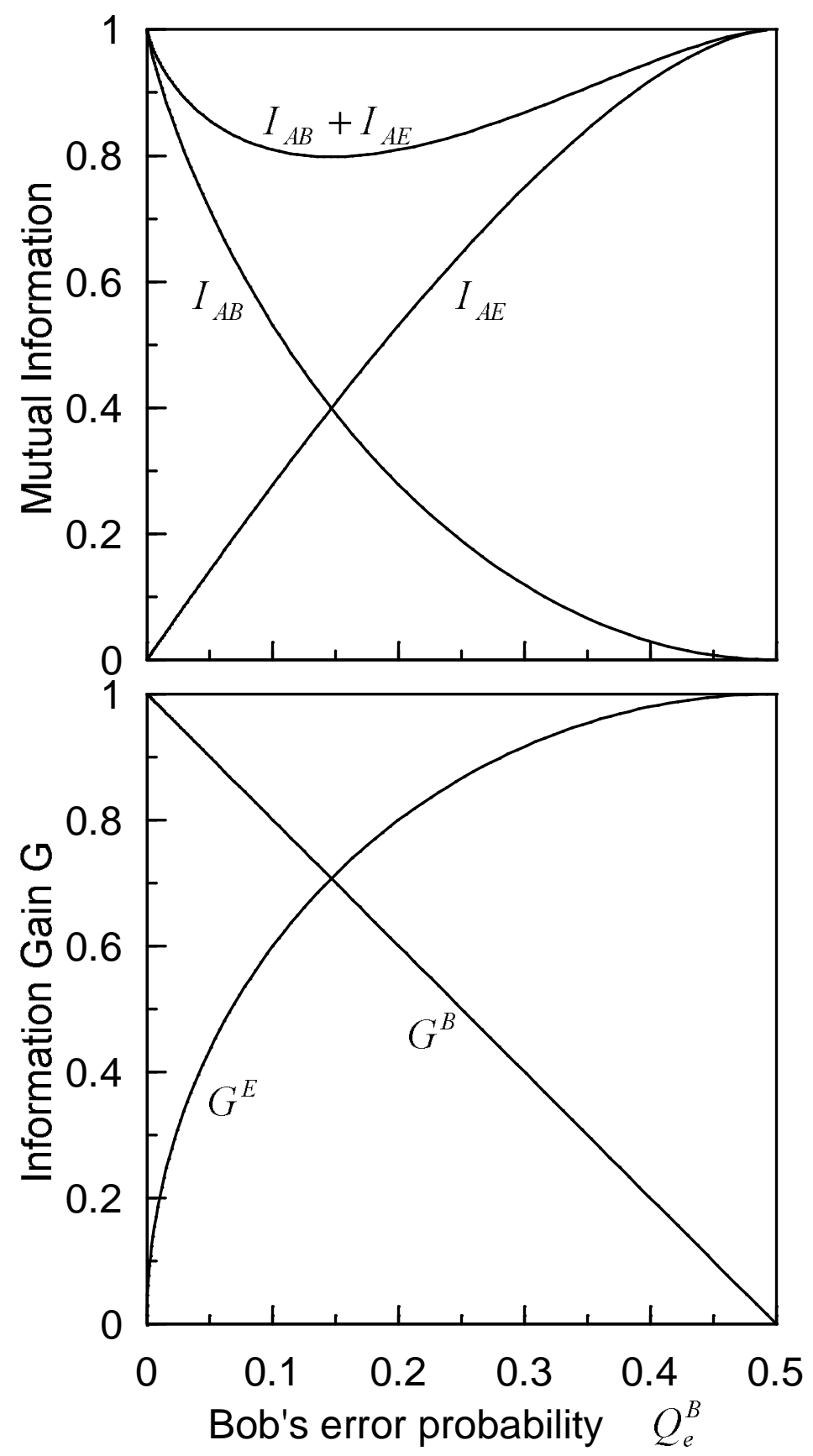

FIG. 1

Y. Nambu et al., "Quantum Key Distribution Using Two Coherent States ..." 
Fock space $:\{n\rangle: n \in I\}$

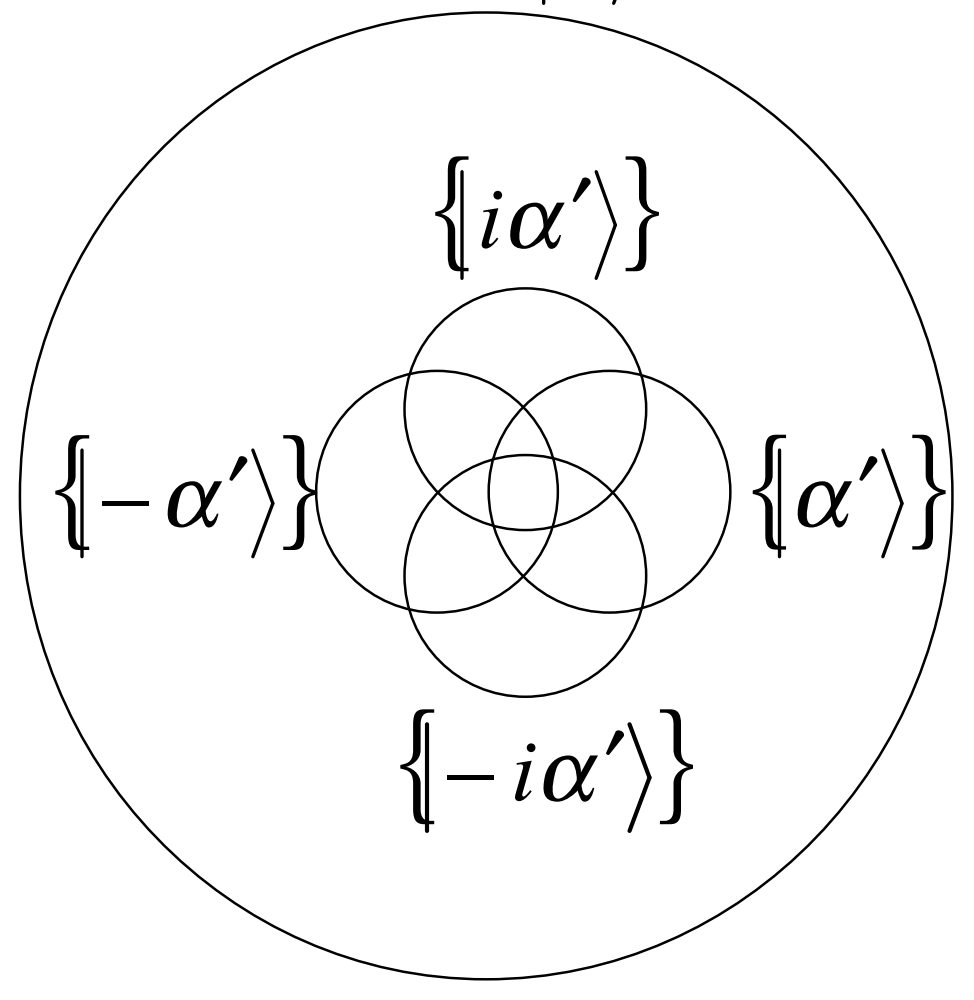

FIG. 2

Y. Nambu et al., "Quantum Key Distribution Using Two Coherent States ..." 


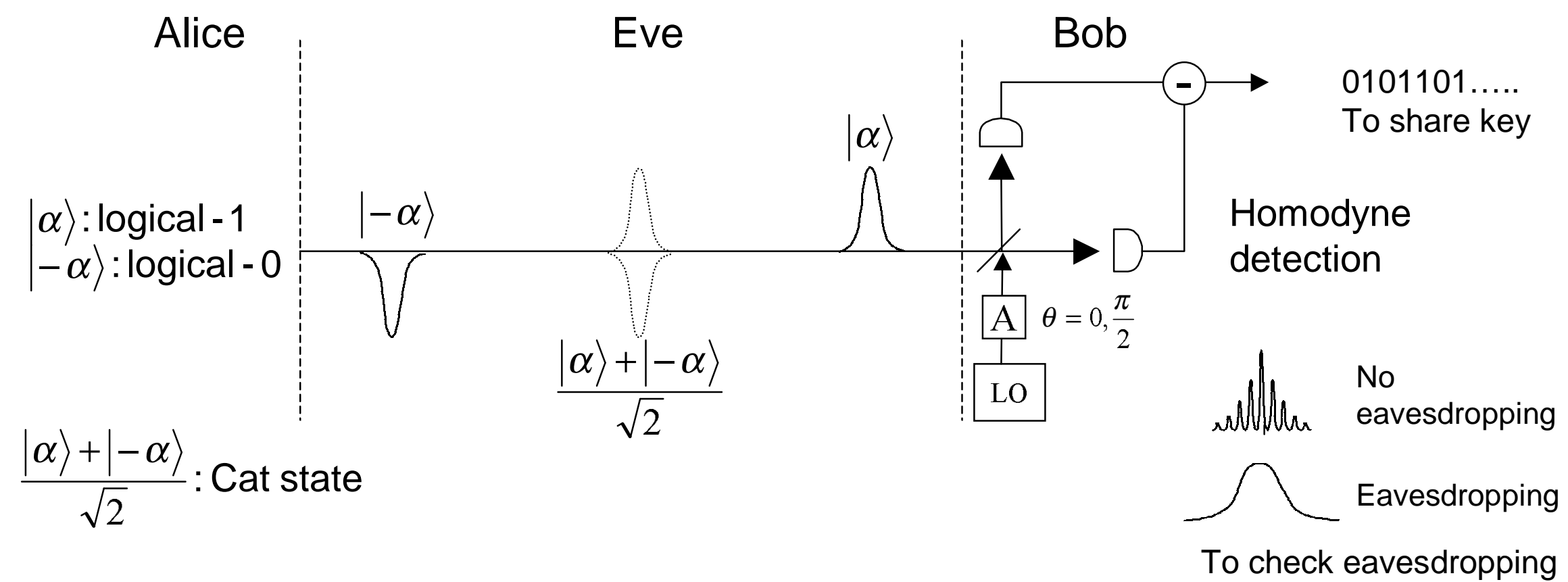

FIG. 3

Y. Nambu et al., "Quantum Key Distribution Using Two Coherent States ..." 


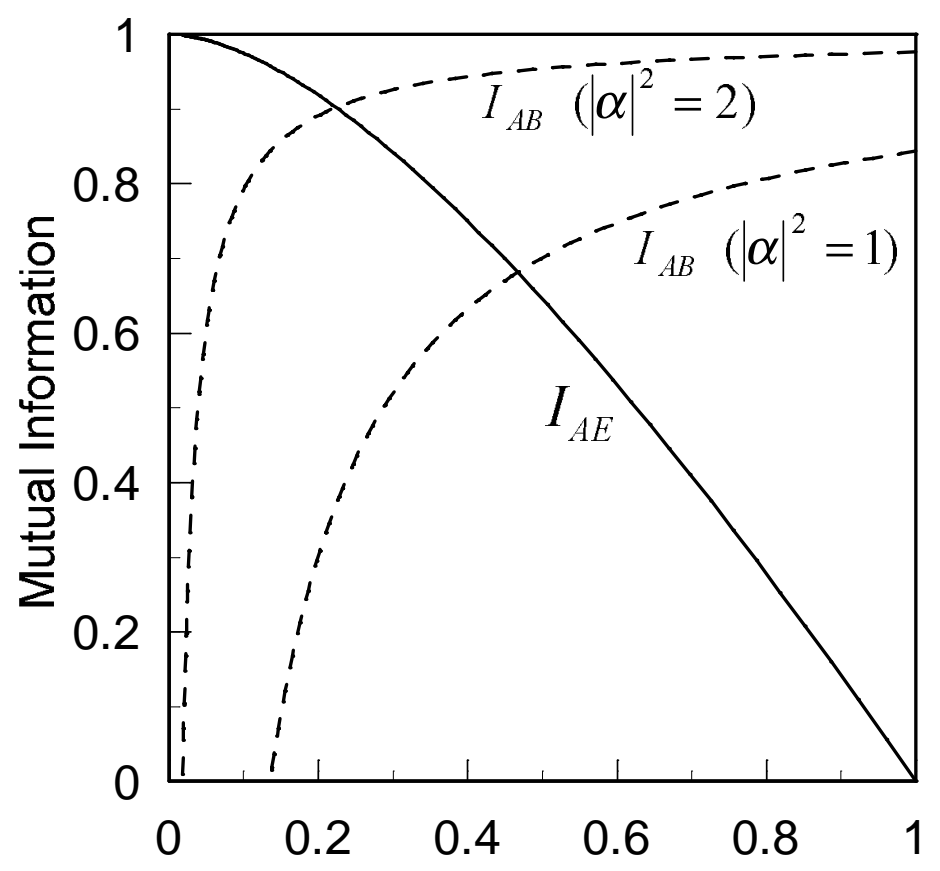

Fringe visibility

FIG. 4

Y. Nambu et al., "Quantum Key Distribution Using Two Coherent States ..." 